\title{
Geschäftsbericht 2020 der FMH Services Genossenschaft
}

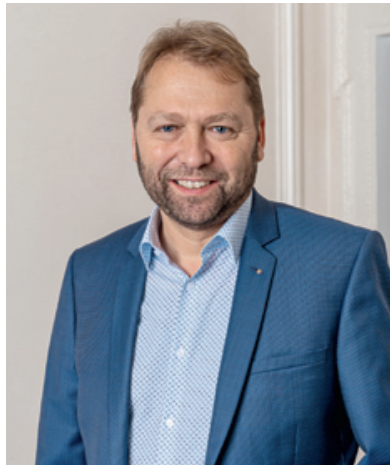

Dr. med., MLaw Beat Bumbacher Präsident der Verwaltung FMH Services Genossenschaft

Die FMH Services Genossenschaft hat 2020 ein herausforderndes und anspruchsvolles Jahr hinter sich. Die Finanzanlagen erholten sich nach anfänglich deutlichen Marktverwerfungen und schlossen mit einem leichten Zuwachs. Trotzdem steht dem ein leichtes Minus im Gesamtergebnis gegenüber bei positivem Ausblick. Die Substanz der FMH Services Genossenschaft ist unverändert gesund. Der Geschäftsbericht 2020 kann auf unserer Website www.fmhservices.ch eingesehen werden.

Die im operativen Bereich tätige FMH Consulting Services AG hat 2020 gut gearbeitet. Die Nachfrage nach unseren Dienstleistungen ist unverändert hoch. Das Team von FMH Services verfügt über fundiertes Fachwissen und grosse Projekterfahrung. Mit fast 100 Jahren Knowhow im Gesundheitswesen wissen wir, was unsere Kundinnen und Kunden brauchen, um effizient und erfolgreich zu sein.

Erfolg und Tradition verpflichten. So ist es unser Bestreben, Gutes zu bewahren und Neues zu entwickeln. Die laufende Pandemie erfordert auch von der FMH Services Genossenschaft Agilität, um Ihnen weiterhin alles aus einer Hand für den unternehmerischen und administrativen Betriebsalltag anbieten zu können. Wir sind der führende Partner in der Schweiz, wenn es um den Kauf, die Gründung, Führung und Übergabe einer Arztpraxis oder einer anderen ambulanten ärztlichen Institution geht. Es ist mir ein grosses Anliegen, allen Mitarbeiterinnen und Mitarbeitern der FMH Services für ihr grosses und professionelles Engagement ganz herzlich zu danken. Der Dank geht auch an alle Mitglieder in unserem schweizweiten und engmaschigen Partnernetzwerk. Ihnen als Genossenschafterinnen und Genossenschafter danke ich herzlich für Ihre Treue und das Vertrauen, welches wir bei Ihnen geniessen. Wir freuen uns, Ihnen auch weiterhin als verlässlicher Partner mit einer umfassenden Dienstleistungspalette zur Verfügung zu stehen.

\section{Rapport de gestion 2020 de la coopérative FMH Services}

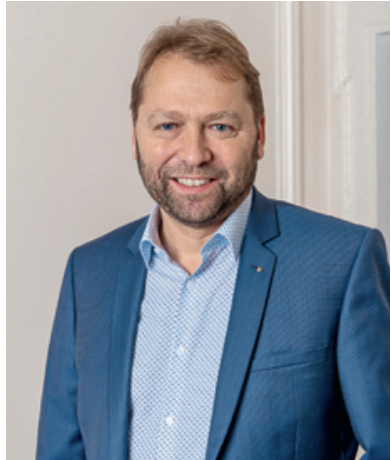

Dr méd. Beat Bumbacher, MLaw, président du conseil d'administration de la coopérative FMH Services

La coopérative FMH Services a connu une année 2020 difficile et riche en défis. Après de fortes turbulences sur les marchés financiers, les placements financiers se sont redressés et ont clôturé sur une légère augmentation. Malgré cela, le résultat global présente un petit déficit qui s'inscrit dans des perspectives positives. La santé financière de la coopérative FMH Services reste toutefois intacte. Le rapport de gestion 2020 peut être consulté sur notre site www.fmhservices.ch.

FMH Consulting Services AG active dans le domaine opérationnel a très bien travaillé en 2020. La demande pour nos prestations reste élevée. L'équipe de FMH Services dispose de connaissances solides et d'une vaste expérience dans la gestion de projets. Avec près de 100 ans de savoir-faire dans le domaine de la santé, nous savons ce dont nos clientes et clients ont besoin pour travailler avec efficacité et succès.

Succès et tradition obligent. Nous nous efforçons donc de préserver le meilleur tout en restant innovants. Dans la pandémie actuelle, la coopérative FMH Services doit aussi faire preuve d'agilité pour pouvoir continuer à vous proposer un interlocuteur unique qui sache répondre à vos questions entrepreneuriales et administratives quotidiennes. Nous sommes le partenaire leader en Suisse quand il s'agit de l'acquisition, de la fondation, de la gestion et de la remise d'un cabinet médical ou d'une autre institution médicale ambulatoire. Je tiens à adresser mes chaleureux remerciements à tous les collaborateurs et collaboratrices de FMH Services pour leur engagement professionnel et sans faille. Mes remerciements vont aussi à tous les membres de notre réseau de partenaires actifs dans toute la Suisse.

Quant à vous, chères et chers sociétaires, je vous remercie vivement de votre fidélité et de votre confiance. Nous nous réjouissons de pouvoir à l'avenir aussi vous apporter un soutien fiable avec un large éventail de prestations. 